\title{
Elaboración del marco teórico en la formación universitaria docente: algunas claves para comprender sus implicaciones
}

\author{
Gustavo Toledo Lara* \\ Universidad Camilo José Cela, Madrid, España \\ https://orcid.org/0000-0002-5104-9555
}

\section{Resumen}

En este artículo se presenta un análisis cualitativo sobre las posibles implicaciones de la elaboración del marco teórico en la formación universitaria docente. A partir de la exploración teórica, se identifica la necesidad de reconocer el contexto en el que se desarrolla la experiencia investigativa de los estudiantes, además de reconocer y diferenciar los elementos constitutivos del marco teórico. Todo lo anterior debe estar sustentado en el fomento del pensamiento crítico y la capacidad argumental desde el inicio de los estudios universitarios con el objetivo de asumir la capacidad crítica y reflexiva de forma que se pueda extrapolar a la futura acción docente en las aulas. La acción tutorial, la hipótesis de progresión y el diseño curricular deben permitir el establecimiento de estrategias que mejoren la atención personalizada de los estudiantes una vez que inician su experiencia investigativa. Finalmente, resulta determinante la formación tanto para el docente que orienta a los futuros educadores, como para los propios estudiantes, ya que la motivación y el fortalecimiento de la vocación docente constituyen elementos vinculantes al momento de integrar las competencias propias de los profesionales del siglo XXI y más al tratarse de la disciplina docente como campo de estudio y de formación.

Palabras clave: enseñanza universitaria; formación docente; pensamiento crítico; análisis teórico; investigación educativa

\section{Development of the Theoretical framework for university training on education: some clues to understand its implications}

\begin{abstract}
This article presents a qualitative analysis regarding the possible implications of the development of the theoretical framework for university training on education. On the basis of the theoretical research, the need to recognize the context in which the students' research experience takes place is identified, apart from recognizing and differentiating the components of the theoretical framework. All of the above should be based on the promotion of critical thinking and argumentative capacity from the beginning of the university studies in order to develop a critical and reflective capacity so that it can be extrapolated in the future teaching action in the classroom. The tutorial action, the hypothesis of progression and the curriculum design must allow the establishment of strategies that improve the personalized attention of the students once they begin their research experience. Finally, training is crucial both for the teacher who guides the future educators and for the students themselves, since motivation and the strengthening of the teaching vocation are binding elements when
\end{abstract}


it comes to integrating the skills pertaining to the professionals of the twenty-first century, more so when it comes to the teaching discipline as a field of study and training.

Keywords: University teaching; teacher training; critical thinking; theoretical analysis; educational research

\title{
Elaboração do marco teórico na formação universitária docente: algumas chaves para compreender suas implicações
}

\begin{abstract}
Resumo
Neste artigo, apresenta-se uma análise qualitativa sobre as possíveis implicações ao se elaborar o marco teórico na formação universitária docente. A partir da exploração teórica, identifica-se a necessidade de reconhecer o contexto em que se desenvolve a experiência de pesquisa dos estudantes, além de reconhecer e diferenciar os elementos constitutivos do marco teórico. O supracitado deve-se sustentar no fomento do pensamento crítico e a capacidade argumentativa desde o início dos estudos universitários com o objetivo de assumir a capacidade crítica e reflexiva e, assim, extrapolar a futura ação docente nas aulas. A ação tutorial, a hipótese de progressão e o desenho curricular devem permitir o estabelecimento de estratégias que melhorem a atenção personalizada dos estudantes quando iniciarem atividades de pesquisa. Finalmente, a formação é determinante tanto para o docente que orienta os futuros educadores, quanto para os próprios estudantes, visto que a motivação e o fortalecimento da vocação docente constituem elementos vinculantes no momento de integrar as competências próprias dos profissionais do século XXI e, mais ainda, ao tratar a disciplina docente como um campo de estudo e de formação.
\end{abstract}

Palavras-chaves: ensino universitário; formação docente; pensamento crítico; análise teórica; pesquisa educativa prender sus implicaciones. Revista Digital de Investigación en Docencia Universitaria, 13(2), 53-66: https://doi. org/10.19083/ridu.2019.1083

E l conjunto de competencias que han de adquirirse en la educación superior deben permitir que, en efecto, la superación de los deberes propios pueda realizarse, es decir, al cabo de los estudios se espera que el cúmulo de experiencias posibilite realizar la síntesis de lo aprendido (Serrano \& Pontes, 2015). Todo ello se traduce en la elaboración de un trabajo final. Ahora bien, desde un punto de vista metodológico ese trabajo debe contener una serie de elementos que, de forma armónica, organizada y metodológica, puedan transmitir algunos hallazgos y en su caso, la aplicabilidad de alguna propuesta.

En el contexto de la formación docente la realidad no es diferente, solo que, la connotación docente de esa formación debe permitir que, en este caso, se pueda hacer un abordaje teórico desde el análisis, la síntesis de la información, la argumentación y el pensamiento crítico (Amarillo, Corredor, \& Ávila, 2016; Mero, 2019). Ante esta realidad son diversos los aportes teóricos que exponen críticas al modelo actual de aprendizaje a nivel universitario, el cual no parece favorecer eficazmente este tipo de competencias (Bezanilla-Albisua, Poblete-Ruiz, Fernández-Nogueira, Arranz-Turnes, \& Campo-Carrasco, 2018; Palacios, Álvarez, Moreira, \& Morán, 2017; Vidal-Moscoso \& Manríquez-López, 2016).

Ahora bien, ¿El marco teórico es un problema? Si entendemos este aspecto como una investigación en sí misma y que además necesita de lectura analítica, comparación de aportes teóricos, o de argumentos que puedan explicar la corresponden- 
cia de ese marco teórico con el tema central del trabajo, al parecer sí que es un problema. Esta práctica no siempre está presente a lo largo de los estudios y es justamente al final de estos cuando se descubre la existencia y formalidad de este tipo de trabajos (Moyano, 2018; Yubero \& Larrañaga, 2015).

También, y retomando el contexto de la formación docente, puede resultar un problema, pues existe una tendencia a asociar esta formación casi de forma exclusiva al aspecto del trabajo en el aula, es decir, al aprendizaje de la práctica docente, obviando así que, si se pretende que la educación forme a ciudadanos en pensamiento crítico, analítico e interpretativo, lo adecuado sería formar a los docentes en este tipo de competencias (Yubero \& Larrañaga, 2015). De forma que, al llegar al momento de plasmar en un trabajo el fruto de este proceso crítico, este no resulte desconocido.

El sentido utilitarista e inmediatista de lo que se aprende va en contra precisamente del aspecto antes señalado, ya que se prioriza la adquisición de herramientas que necesitan ser aplicadas directamente en el aula y la capacidad de análisis o de pensamiento crítico no suele ser considerada como de aplicación directa (Santibáñez, 2014). No se visualiza esta capacidad como necesaria para superar los estudios de formación docente y menos como un elemento imprescindible en la práctica. De allí, se establece como objetivo principal de este análisis, la exploración de las connotaciones e implicaciones de la elaboración del marco teórico, desde la perspectiva de la formación docente.

Para alcanzar este objetivo, se hace necesario, por una parte, intentar explicar el verdadero sentido del marco teórico en un proceso de investigación científica, y por otro, proponer algunas claves para comprender si en efecto, el proceso metodológico y analítico que acompaña todo marco teórico es propio de la formación docente.

\section{El verdadero sentido del marco teórico en un proceso de investigación científica}

A juicio de Guadarrama (2016) la ciencia no puede seguir su proceso de avance sin la presencia de un marco teórico, aunque este sea frágil en su construcción. Esto significa que se hace más que imperativa la necesidad de contar con un contexto teórico que permita ubicar las teorías que forman parte del objeto de la investigación. En la medida que el marco teórico constituye la columna de toda investigación (Tusco, Quesocala, \& Tito, 2011), aquí confluyen no solo las teorías sino el aporte al conocimiento nuevo como resultado del análisis, la inferencia y la argumentación que se puedan realizar mientras se desarrolla el proceso investigativo.

Si partimos del punto de vista de que se trata de una investigación de corte cualitativo, también estamos de acuerdo en que tiene que ver con un estudio metódico y ordenado en cuyo caso las fuentes de información constituyen la base sobre la que reposa la posible comprensión del problema. Por ejemplo, en la investigación cualitativa se habla de fenomenología en la cual se "busca conocer los significados que los individuos dan a su experiencia y lo importante es aprender el proceso de interpretación por el que la gente define su mundo y actúa en consecuencia" (Guerrero, 2016, p. 5). Además, en la fenomenología "se insiste mucho en el papel de la intencionalidad humana y en la primacía de la experiencia subjetiva inmediata" (Guadarrama, 2016, p. 149).

En este tipo de investigación, se justifica principalmente la existencia de un marco teórico sumamente denso, completo y complejo en el que el aspecto descriptivo apenas ocupa un lugar, mientras que el aspecto argumentativo, crítico e interpretativo adquiere singular importancia, ya que es la faceta más característica que, en este caso, se espera de este tipo de investigación (Risco del Valle, 2018). De forma tal, el marco teórico, que es una investigación propiamente dicha, va a servir de encrucijada de saberes y de teorías. Es el propio investigador el que va a integrar en su hilo discursivo y argumental todo lo que ha podido investigar, revisar y procesar.

En este caso, se debe tener en cuenta que en el marco teórico existe un fuerte riesgo de dispersión argumental. Esto significa que el argumento que se produce de parte del propio investigador puede llegar a desviarse, lo que ocasiona así que el aporte del marco teórico sea una adición desvinculada de subtemas o elementos que se cree que son propios del tema objeto de estudio.

Para evitar la dispersión argumental, se hace necesario establecer una serie de categorías de análisis que permitan ubicar y condensar tanto la 
discusión como la interpretación, de forma que el texto que se genere no se pierda en explicaciones o detalles no siempre relevantes o vinculantes a la investigación. Por lo tanto, y desde la investigación educativa como contexto, siempre va a resultar necesario determinar el método para llevar a cabo el análisis sin obviar el hecho de que los resultados obtenidos deben obedecer a un conjunto de construcciones de orden teórico con el objeto de que ayuden a comprender el tema objeto de estudio (San Martín, 2014).

El sentido del marco teórico pasa por el reconocimiento de las etapas fundamentales que se vinculan con su desarrollo. Resulta poco práctico el hecho de plasmar en un texto un conjunto de ideas en el que no se observe un recorrido previo proveniente de la exploración de los aportes teóricos resultantes de la investigación. En este orden de ideas, el llamado estado del arte se ubica como primer paso en la investigación, ya que de allí se nutre el marco teórico, por lo tanto, es un aspecto de importancia determinante en el momento de desarrollar la indagación propiamente dicha (Guevara, 2016).

En el desarrollo del marco teórico se pueden encontrar una serie de aristas que, si bien están vinculadas, tienen diferencias en cuanto al sentido de estas dentro de dicho marco. Hablamos entonces de estado del arte, revisión documental, y marco teórico. El estado del arte persigue exponer un conjunto de propuestas teóricas y metodológicas sobre el objeto de estudio, que es lo que se está investigando, con lo cual, se muestra la discusión actual de dicho tema (Gómez, Galeano, \& Jaramillo, 2015). Al hablar de revisión documental se trata de aquella exploración que se hace tanto de textos publicados como de experiencias de corte investigativo que se han desarrollado en torno al objeto de estudio (Guevara, 2016). En relación con el marco teórico, se hace necesario reafirmar la idea de que este busca comunicar y compartir el resultado crítico y argumentado de textos especializados vinculados directamente con el contexto de la investigación que se desarrolla (Herrera, Guevara, \& Munster, 2015).

Otra arista importante en el marco teórico es el conocido como marco conceptual. De forma errónea se tiende a confundir el marco teórico con el marco conceptual, ya que, al observar el texto fi- nal del trabajo, se pueden entrelazar los conceptos con el desarrollo teórico y argumental (Tusco et al., 2011). Sin embargo, el marco conceptual en ningún caso es una compilación de conceptos, es decir, implica una relación teórica que va a servir precisamente para que el investigador determine cuáles son los instrumentos básicos para la comprensión del fenómeno que está investigando (conceptos) con el objeto principal de establecer lo que, para la investigación, se asume desde un concepto determinado.

Por lo tanto, en todo marco teórico se hace necesaria la precisión conceptual y a partir de esta, han de quedar correctamente establecidos los contenidos teóricos de forma que este pueda contribuir de alguna manera a ubicar el problema que se está investigando y su relevancia al campo o área de conocimiento correspondiente.

Es de reconocer que, al menos en la generalidad de los ejercicios investigativos contextualizados en la educación, se tiende a englobar el marco teórico a partir de la integración de las aristas antes señaladas. Evidentemente estos elementos van a experimentar modificaciones y adaptaciones en función del tipo de investigación y del nivel de estudios. Es decir, la densidad y complejidad del marco teórico no solo estará determinada por el tema objeto de estudio y su naturaleza, sino que conforme se avance en las etapas formativas (grados, másteres o doctorados) este marco podrá incluir otros elementos siempre que estos respondan a los objetivos de la investigación y, en definitiva, que supongan un aporte significativo al desarrollo del trabajo.

Al momento de construir un marco teórico, es necesario tener en cuenta que, como parte del proceso de una investigación, debemos entenderlo como una progresión de fases metodológicas las cuales pueden diferenciarse conforme se avanza en el proceso investigativo. Esa progresión de fases no es lineal, es decir, se acepta que el proceso investigativo es procesual y gradual, pero no es un camino que se recorre en línea recta (Di Marco, 2015).

En relación con las partes que han de configurar el marco teórico, estas obedecen a la necesidad de estructurar de la mejor manera dicho marco con el objeto de que su contenido sea no solo relevante para la investigación, sino que se pueda 
mostrar que el investigador cuenta con las competencias necesarias para organizar la información, expresar el resultado de análisis y el proceso crítico y reflexivo y la posibilidad de dar cuenta de la investigación que se ha realizado sobre el tema objeto de estudio.

De forma tal que se ha de superar la tendencia a caer en lo descriptivo y progresar en cuanto a lo analítico-interpretativo (Palacios et al., 2017). Si bien es cierto que dependiendo del objeto de estudio se podrían presentar un conjunto de características, las mismas no han de convertirse en una adición de elementos descriptivos, sino que deben formar parte del desarrollo argumental del marco teórico.

Hay que señalar que no existe un consenso respecto a las partes que deben conformar el marco teórico. Esta ausencia es el resultado de la multiplicidad de visiones que pueden existir en torno a la metodología de la investigación y evidentemente, a la naturaleza propia de lo que se está investigando. Se puede inferir entonces que el marco teórico se compone de teorías que dan cuenta de los resultados alcanzados en la investigación, con el objeto de que el problema pueda definirse desde una o varias teorías o corrientes ideológicas $\mathrm{y}$, en definitiva, pueda ayudar al investigador a no desviarse del planteamiento originario del problema u objeto de estudio (Rivadeneira, 2017; Tusco et al., 2011). Por su parte, Maya (2014) expone que en el marco teórico deben estar presentes los siguientes elementos: 1.-enfoques teóricos como fundamento del problema objeto de la investigación, 2.-conjunto de observaciones o relación de experiencias sobre el tema objeto de la investigación, y 3.-exploración bibliográfica vinculada al tema objeto de la investigación.

Todo lo anterior, debe acompañarse de dos niveles de trabajo que consisten en: 1.-el ejercicio de revisión y exploración de teorías vinculadas al tema y 2.-la generación de conceptos a partir de la obtención de información sobre la realidad que se está estudiando. Esto último supone tanto las definiciones conceptuales (conceptos extraídos de la lectura y análisis) como las definiciones operacionales (conceptos resultantes de la adaptación a partir de otros conceptos conocidos en función del trabajo).

A partir de las líneas anteriores, puede inferirse que la elaboración del marco teórico es una labor sumamente compleja y difícil. En efecto lo será en cuanto no se adquiera desde el inicio de los estudios el hábito de la lectura y se ejercite la reflexión crítica argumentada. Sin restar importancia al resto de las partes que conforman el informe de investigación (llámese Trabajo de Fin de Grado, Trabajo de Fin de Máster, o Tesis Doctoral), siempre se suele ver el marco teórico como el elemento más incomprendido no solo por ciertas costumbres generalizadas entre estudiantes, sino por el temor a descender en un pozo sin fondo en el que no se ve un panorama claro imprime al marco teórico de un sentido de incertidumbre y hasta rechazo en muchos casos. Esta visión se ve favorecida por la presión de terminar pronto, en el menor tiempo y con lo mínimo aceptable.

Posiblemente, sea necesario redefinir la concepción del docente y empezar a mirarlo como un profesional transformador activo del hecho educativo (Martínez, 2016). Para llegar a este punto, se precisa de un proceso sistemático y progresivo de educar en pensamiento crítico, sin dejar a un lado la idea de que el aprendizaje y la calidad de este tendrá en el docente una de las variables más influyentes en cuanto a su actuación y su posible impacto (Rivero \& Porlán, 2017).

\section{El análisis y la capacidad de reflexión crítica desde la formación docente}

A juicio de Martínez y Márquez (2014) el tema de la formación y desarrollo del conjunto de habilidades investigativas en los estudios universitarios se erige como un aspecto que se hace presente en una cantidad importante de investigaciones educativas, con lo cual, se puede inferir que el análisis y la capacidad de reflexión crítica también es una necesidad dentro de lo que se entiende como formación docente. Esa formación docente se desarrolla a nivel universitario.

Dentro de la formación universitaria se puede identificar los componentes que forman el entramado curricular que pretende organizar este tipo de enseñanzas. Ese entramado debe ir casado con las competencias formativas y profesionales que se esperan alcanzar por el futuro profesional de la educación (Caneiro, 2015).

En este sentido, la responsabilidad de formar a 
la persona que tendrá el deber de formar a otros seres humanos no es un asunto baladí, ya que, sin pretender repetir expresiones comúnmente popularizadas, es cierto que la educación es un pilar fundamental en el desarrollo de las sociedades. Es más, se afirma que la educación es un proceso que se desarrolla en sociedad y que, en efecto ejerce influencia en una formación global que ha de orientarse con miras a que el propio estudiante sea un actor activo de su propio proceso pedagógico. En este sentido, se hace necesario que los estudiantes adquieran habilidades y competencias que les permitan hacer frente a una variedad de actividades de corte formativo para las cuales, se han de apoyar desde la capacidad resolutiva y la autonomía sin obviar que la educación también debe fomentar los valores democráticos, morales y éticos dentro de un contexto de ciudadanía responsable (Moreno-Pinado \& Velázquez, 2017).

Ahora bien, si el docente está llamado a ser una referencia en cuanto al uso del lenguaje, capacidad analítica, comprensión crítica o uso didáctico de las nuevas tecnologías, el Estado debe garantizar los medios para llegar a tal fin. Todo lo anterior indiscutiblemente supone el cuidado en todo lo que tiene que ver con la formación docente e indudablemente los docentes encargados de formar deben igualmente estar formados (Martínez, 2016). Pareciera ser una suerte de relación simbiótica en la cual, tanto formadores como futuros docentes se congregan en el espacio universitario con el objeto de sentar las bases de una formación profesional lo más completa posible.

Dentro de esta formación docente se identifica además la necesidad de desarrollo de un hábito reflexivo, la construcción de conocimientos y la posibilidad de ofrecer aportes que pretendan ser innovadores. En la actualidad, intentar convertirse en docente supone la adquisición de una gama diversa y compleja tanto de destrezas como de cualidades en el contexto social, profesional y humano (Serrano \& Pontes, 2015). Esto incluye, por ejemplo, las competencias que se relacionan con las capacidades pedagógicas, capacidad reflexiva y la capacidad analítica y argumental (Palacios et al, 2017; Risco del Valle, 2018).

En este caso, hablamos entonces de la llamada habilidad investigativa, la cual tiene que ver con aquella acción que se ejecuta con el propósito de superar progresivamente un conjunto de tareas de corte investigativo en el contexto docente o laboral, contando efectivamente con las herramientas que ofrece la metodología de la ciencia. (Fernández \& Villavicencio, 2017; Martínez \& Márquez, 2014).

Esto tendrá una serie de consecuencias de orden pedagógico y didáctico, ya que la formación para la investigación implica una gama de acciones concebidas para facilitar el impulso y la generación de conocimientos, actitudes y habilidades requeridas por parte de los estudiantes, de forma que se pueda finalmente asegurar un óptimo desempeño en la ejecución de las acciones vinculadas directamente con la investigación científica (Guerrero, 2007). La habilidad investigativa cuenta con dos aristas que engloban su acción (Quevedo, García, \& Cañizares, 2018): 1.-la habilidad de base investigativa (relacionada con aquellas habilidades genéricas que sostienen el conocimiento contemporáneo) y 2.-la habilidad de creación investigativa (vinculada con aquellas habilidades de búsqueda y generación de nuevo conocimiento).

De acuerdo con Moreno-Pinado y Velázquez (2017), en muchos casos los estudiantes no tienen la capacidad de analizar los problemas ni de asumir una posición reflexiva al aprender, con lo cual se desemboca en un pensamiento reproductivo con un mínimo esfuerzo mental y a nivel global, un nivel bajo en cuanto al desarrollo del pensamiento crítico. Otro hecho que sigue apareciendo al momento de emprender este tipo de actividades investigativas y al intentar plasmar por escrito el resultado del análisis y la reflexión crítica es el llamado plagio, no obstante, ese plagio generalmente se observa desde el fallo del estudiante y no como un elemento que necesite revisión de toda la comunidad universitaria (Ochoa \& Cueva 2014).

Esto determinará que la experiencia de aprendizaje al final de los estudios quizás no resulte tan satisfactoria debido a la sensación de incertidumbre que puede llegar a generar el querer expresar y argumentar con cierto nivel. Evidentemente se ha de contar y hasta esperar que los estudiantes tengan cierto grado de autonomía e iniciativa por sus estudios además de tener un acompañamiento adecuado, no obstante, y aun contando con lo anterior, aquella competencia crítica, analítica y 
reflexiva la cual ha de expresarse en este caso en el marco teórico, puede no resultar familiar en muchos casos.

Entendiendo como pensamiento crítico aquel proceso de orden intelectual que se dinamiza desde el momento en el que la información se procesa, analiza e interpreta desembocando en un nuevo conocimiento que ha de aplicarse en el mundo real (Moreno-Pinado \& Velázquez, 2017), hay una serie de consideraciones que han de tomarse en cuenta dentro de este tema de la formación docente. Las corrientes reformistas universitarias coinciden en la mayoría de los casos en la necesidad de formar ciudadanos bajo ciertas competencias y habilidades que les permitan dar el paso para incursionar y participar en el desarrollo de las sociedades, con lo cual, se rescata la idea de que se pueda aplicar en el mundo real lo que se ha aprendido a lo largo de los años de estudio (Fernández \& Villavicencio, 2017; Morales-Gómez, Arteaga-Rolando, Gallegos-Samaniego, Yanchapaxi-Sánchez, \& Stay-Zúñiga, 2016). Evidentemente, esto incluye la formación docente.

El detalle está en que no siempre se favorece en este caso el pensamiento crítico, sino que se fomenta directa o indirectamente, la idea de que el conocimiento disciplinar y la memorización de técnicas de aplicación directa en el aula es lo que se espera de que una persona debe aprender para ser docente. Esto, en desventaja ante la visión de un docente como investigador de su propia práctica (Davini, 2015), o como práctico reflexivo (Carbonell, 2017). Por lo tanto, se puede asegurar que desde la pedagogía crítica se rechaza aquel rol docente como agente transmisor de ideas ajenas haciendo a un lado la problematización del hecho pedagógico y, por ende, la ausencia del fomento del pensamiento crítico (Tamayo, Zona, \& Loaiza, 2015).

Dicho esto, se hace necesario reconocer que la formación del pensamiento crítico va a necesitar un conjunto de habilidades que giran en torno al análisis, la interpretación, la evaluación y la inferencia (Moreno-Pinado \& Velázquez, 2017). Todo esto acompañado de una cosmovisión que favorezca la construcción de un criterio propio por parte del estudiante o lo que es lo mismo, que el estudiante pueda ir construyendo progresivamente una posición crítica y fundamentada que le permita elaborar inferencias, explicarse y en su caso, proponer soluciones. Hablamos entonces de que, desde la universidad y dentro del contexto de la formación docente se han de favorecer iniciativas compatibles con la puesta en práctica de estrategias metacognitivas, lo cual pasa por la concepción problematizadora de aquello que se está estudiando e investigando.

La acción tutorial en la formación docente es una responsabilidad que se comparte entre el estudiante y el director o tutor, en este caso, del proceso investigativo y su desarrollo (Quevedo et al., 2018). Retomando la idea de las connotaciones derivadas del momento en que se tiene que desarrollar un marco teórico, dadas las consideraciones anteriores, la acción tutorial tiene un contexto particular, ya que al menos en teoría es un docente experimentado que está formando a un futuro docente en cuanto al desarrollo de su ejercicio investigativo. Hasta este punto, posiblemente exista un consenso generalizado. No obstante, esta acción cuenta con una serie de implicaciones que van a determinar la progresión de esa experiencia de análisis crítico para lo cual, parece ser útil retomar los saberes previos y la evolución del estudiante (Aguilar-Parra, Alías-García, Álvarez, Fernández-Campoy, Pérez-Gallardo, \& Hernández, 2015; Caneiro, 2015).

El objeto de este trabajo no es el estudio de la acción tutorial, sin embargo, este concepto es determinante al momento de iniciar el proceso investigativo. Se hace especial mención a la elaboración del marco teórico y sus implicaciones vistas desde la formación docente debido a que, en esa parte tan característica de toda investigación, entra en juego un cúmulo de factores que van, desde la progresión de los estudios, el desempeño estudiantil y académico, hasta el posible impacto de la acción tutorial en el desarrollo de la investigación por parte del estudiante (Venegas-Ramos \& Gairín, 2018). Es decir, esta acción tutorial fomenta una vinculación de dependencia porque el investigador que se inicia requiere de orientación en muchas facetas, con lo cual el tutor o director se erige como un agente orientador y regulador de la ejecución de actividades de corte científico (Quevedo et al., 2018).

La acción tutorial no se limita solamente a la superación de pasos dentro de la formalidad investigativa, sino que además la acción tutorial 
no debe dejar de considerar que la habilidad de interacción entre el tutor o director y tutorado va a permitir el reconocimiento de la experticia, la formación y la disposición para enseñar por parte del tutor por un lado, y por otro, se va a crear una atmósfera formativa en la que el tutor podrá ir formando al estudiante a partir de la observación sistemática que se haga sobre este al momento de enfrentarse a los retos propios de todo inicio en cuanto a una experiencia de corte investigativo (Ochoa \& Cueva, 2014).

Desde un punto de vista pedagógico, la acción tutorial si bien es cierto que a nivel global implica orientación y acompañamiento, también es un ejercicio por parte del tutor o director en cuanto al reconocimiento de las condiciones y experiencias que tiene el estudiante una vez que inicia su propia experiencia investigadora (Alcívar \& Gorozabel, 2018). En este sentido, tanto la motivación como las características de cada estudiante se convierten en aspectos clave (Venegas-Ramos \& Gairín, 2018). Por cuanto llegan a determinar no solo la forma en que se inicia el proceso de análisis y desarrollo de argumentos propios, en este caso del marco teórico, sino que la motivación y la disposición puede facilitar o entorpecer.

Ante este posible escenario se habla de la llamada hipótesis de progresión (HP) (García, Porlán, \& Navarro, 2017). Esta hipótesis consiste en el reconocimiento de los niveles de partida en el que se encuentran los estudiantes cuando se enfrentan por primera vez a los aspectos que han de dar respuesta. Este reconocimiento se basa en la identificación de los saberes previos, referencias personales, experiencias estudiantiles y recorrido académico (Ramírez-Casallas, 2018). Al establecer la hipótesis de progresión se intenta personalizar la acción tutorial de forma que en el mismo proceso tutorial se encuentren de forma indivisible tanto la particularidad de cada estudiante, el objetivo que se pretenda alcanzar, y las posibles limitaciones que se deban trabajar con el fin de que lo progresivo, sistemático y dinámico esté presente desde el inicio (Espinel \& Valbuena, 2017).

Lógicamente se entiende que todo proceso investigativo no se desarrolla de forma lineal, y en el caso del marco teórico la visión analítica y la construcción de argumentos fundamentados requiere de forma especial un seguimiento y acompaña- miento, para que el esfuerzo no se diluya en aspectos poco o nada vinculantes con el tema objeto de estudio. Por lo tanto, las hipótesis de progresión nos van a ayudar a identificar en los estados de complejidad progresivos, aquellos modelos que se encuentran en la mente de los estudiantes, mientras que nos van a permitir construir un conjunto de actividades secuenciadas con el fin de reducir aquellos elementos que puedan impedir en su caso, que se produzca el aprendizaje (García, Porlán, \& Navarro, 2017).

\section{Esferas que han de considerarse para posibilitar el análisis crítico en el contexto formativo de los estudiantes universitarios}

Al establecer algunos criterios orientadores de cara a la mejora de la experiencia de los estudiantes con respecto a la elaboración de actividades que tienen que ver con el fomento del pensamiento crítico, la capacidad argumental y la reflexión, es fundamental tener en cuenta que se trata de un proceso gradual y progresivo (Santibáñez, 2014). Esta particularidad ha de verse desde varias esferas que, al estar relacionadas entre sí, deben posibilitar que el contexto formativo de los estudiantes permita que el análisis y la expresión escrita de este, forme parte de la práctica habitual a lo largo de los estudios y que, en este caso, la estamos contextualizando en la redacción del marco teórico.

Si bien es cierto que el marco teórico forma parte de los elementos de los trabajos finales de las titulaciones universitarias, tal y como se ha hecho referencias líneas atrás, no es la única parte que necesita este matiz reflexivo y argumental, solo que, es en este caso que se hace necesario intentar lograr esa síntesis de saberes y competencias acumuladas a lo largo de los estudios que se conjugan en la experiencia investigativa de los estudiantes. Así, identificamos cinco esferas vinculadas al fomento del pensamiento crítico desde la formación docente en el contexto universitario. Esas esferas son: 1.-Esfera universitaria, 2.-Esfera operativa, 3.-Esfera didáctica, 4.-Esfera de la acción tutorial y 5.-Esfera estudiantil.

1. Esfera universitaria: aquí se engloban las políticas institucionales que han de pretender el 
fomento del análisis y la capacidad argumental como estrategia de aprendizaje y, por lo tanto, desde la propia universidad se han de establecer las líneas de acción conducentes principalmente a la reducción de la brecha existente entre los diseños curriculares y aquellas competencias profesionales y sociales esperables en los profesionales (Caneiro, 2015; Ramírez-Casallas, 2018). Por otra parte, se necesitan diseñar planes de formación dirigidos a los docentes universitarios con el objeto de acercarles a nuevas metodologías didácticas en este caso, enfocadas a los estudios universitarios dirigidos a la docencia (Alcívar \& Gorozabel, 2018).

2. Esfera operativa: de forma generalizada se tiende a rechazar sin mayores consideraciones, todo lo que tiene que ver con lo memorístico asociándolo a prácticas nocivas para el aprendizaje (Rodríguez \& Pérez, 2017). Lo memorístico será contraindicado en la medida que sea la única forma de demostrar que se ha aprendido y se ejecuten medidas punitivas para enjuiciar los resultados no esperados. Es decir, si no se repite la lección, se recibe un castigo y lógicamente este proceder ya no debería estar presente en ninguna experiencia de aprendizaje. No obstante, a lo largo de los estudios universitarios sí que debe estar presente la capacidad de memoria por parte de los estudiantes, ya que han de demostrar la habilidad de conjugar lo que tienen en mente con su aplicación en el mundo real, es decir transferencia e inferencia de la información (Amarillo et al., 2016; Vidal-Moscoso \& Manriquez-López,2016).

3. Esfera didáctica: al momento de diseñar las estrategias que pretendan favorecer el aprendizaje de los docentes en formación, se hace imperativa la necesidad de que las actividades tengan un componente que permita el aprendizaje progresivo en cuanto a la capacidad analítica y a la redacción propia de un nivel universitario (Rivero \& Porlán, 2017). No siempre el número elevado de tareas poco significativas va a resultar útil sobre todo cuando los trabajos finales que tienen que realizar los estudiantes universitarios se convierten en experiencias casi desconocidas, ya que a lo largo de los estudios generalmente no se han aprendido técnicas vinculadas a este tipo de actividades (Fernández \& Villavicencio, 2017). Es decir, no se debe esperar hasta el fin próximo de los estudios para que los futuros docentes que se están formando tengan el primer acercamiento con el método científico y sus implicaciones (Rodríguez \& Pérez, 2017).

4. Esfera de la acción tutorial: se entiende que el estudiante universitario, en este caso el estudiante que se prepara a nivel universitario para ejercer la docencia ha de contar con un grado importante de autonomía y responsabilidad ante sus estudios. Sin embargo, también es determinante la acción tutorial que pueda ejercer el tutor en esta, posiblemente primera experiencia investigativa de sus tutorados (Venegas-Ramos \& Gairín, 2018).

Así, no resulta suficiente contar con trayectoria investigativa para saber enseñar a investigar, ya que se necesita una didáctica que se corresponda con las necesidades formativas de los estudiantes (Aguilar-Parra et al., 2015). Aquí se puede, por ejemplo, rescatar la idea de la hipótesis de progresión con el fin de diseñar itinerarios personalizados para cada caso. El respeto a la identidad de la persona también tiene que ver con el reconocimiento de sus fortalezas y sus retos y cómo esto se ha de convertir en una herramienta para la orientación y el acompañamiento (García, Porlán, \& Navarro, 2017).

5. Esfera estudiantil: de entrada, se asume que el estudiante universitario es un adulto y como tal, se espera que esté en la capacidad de asumir la responsabilidad de sus estudios. Esta responsabilidad adquiere un sentido especial cuando nos encontramos dentro de la formación docente (Martínez, 2016). La formación de los docentes es y debe ser uno de los aspectos más cuidados, porque se trata de formar a los que van a formar a otros seres humanos y esto no es algo superfluo. El reconocimiento de los estudios docentes a nivel universitario y la responsabilidad de estos ante la sociedad debe ser una razón de peso cuando se toma la decisión de formarse profesionalmente para la enseñanza sin dejar de reconocer que la voca- 
ción docente es indispensable para asumir este camino por recorrer (Davini, 2015).

La interacción entre los actores del hecho educativo permite el surgimiento de tendencias pedagógicas que, al considerarse emergentes, caracterizan la evolución del quehacer educativo en función ya no del clásico binomio enseñanza-aprendizaje, sino que se trata más bien del interaprendizaje en el que tanto el docente como el estudiante se relacionan de forma elíptica teniendo como pilares tanto la formación (desarrollo humano), como el aprendizaje (desarrollo científico) (Morales-Gómez et al., 2016).

En este sentido, la metodología de la investigación enfocada hacia las primeras experiencias investigativas de los estudiantes va a recibir indudablemente la influencia de las pedagogías emergentes que se están desarrollando actualmente (Morales-Gómez et al., 2016). Se habla entonces de la autogogía (fomento de la libertad, autonomía y personalización del hecho educativo), la hodegogía (el docente como orientador y facilitador de procesos), la neurogogía (importancia de los procesos cognitivos, la formación y el aprendizaje) y la infopedagogía (integrar efectivamente las tecnologías en los procesos formativos). Todo lo anterior supone un proceso paulatino y sistemático que pueda garantizar una adecuación efectiva de los nuevos contextos formativos, con lo cual, la formación didáctica universitaria es fundamental para adaptar el modelo pedagógico universitario a las necesidades reales de la sociedad, ya desde una visión integradora y adaptada a los nuevos escenarios (Martín del Pozo, Pineda, \& Duarte, 2017).

Tal y como se ha venido exponiendo, el pensamiento crítico se posiciona como aspecto relevante no solo para el desarrollo de toda la trayectoria de los estudios universitarios, sino que además supone una herramienta determinante para la aproximación a la dinámica investigadora. Así, se proponen tres estrategias para el fomento de pensamiento crítico en el contexto universitario: el aprendizaje basado en problemas, el estudio de caso y los círculos de reflexión (Gil-Galván, 2018; Gamboa, 2017; García, 2017). Todo esto irá acompañado del respectivo proceso evaluativo del pensamiento crítico aplicando en su caso instrumentos de corte cualitativo o cuantitativo como, por ejem- plo, portafolios, observación directa, discusiones grupales, instrumentos de respuesta abierta o cerrada (Ossa-Cornejo, Palma-Luengo, Lagos-San Martín, Quintana-Abello, \& Díaz-Larenas, 2017).

A nivel global, el aprendizaje basado en problemas pretende que el estudiante desarrolle habilidades y destrezas compatibles con el hecho de indagar, plantear hipótesis y ofrecer soluciones, además de desarrollar el trabajo tanto a nivel grupal como individual. Todo lo anterior desde la visión del desarrollo del futuro profesional en formación (Gil-Galván, 2018). Con respecto al estudio de caso, este también se posiciona como una buena estrategia al momento de trabajar el pensamiento crítico, ya que el análisis a profundidad, la revisión, la reflexión y la búsqueda de respuesta a uno o varios problemas fomentan tanto la indagación como el pensamiento analítico y evidentemente crítico (Gamboa, 2017). No obstante, resulta determinante que los docentes cuenten con una formación idónea de manera que el estudio de caso no tienda a la dispersión argumental, más bien que sin obviar el diálogo y la interpretación, no se pierda el sentido del caso que se está analizando. Respecto a los círculos de reflexión, estos ofrecen un contexto favorable para que los estudiantes puedan socializar tanto sus opiniones como sus cuestionamientos (García, 2017) con lo cual, el conflicto cognitivo y el conflicto sociocognitivo son denominadores comunes en esta estrategia, siempre que se pueda orientar este proceso crítico y reflexivo desde una visión del mundo real.

\section{Conclusión}

A lo largo de este artículo se ha intentado hacer una aproximación sobre los aspectos que juegan un rol determinante al momento del abordaje del método científico, específicamente el marco teórico de la investigación, pero contextualizado desde la formación docente ¿Por qué desde la formación docente?

Aunque parezca obvio, la capacidad argumental y analítica debe estar presente en toda formación de nivel universitario. Sin embargo, en la formación docente esta capacidad debe asumirse desde el principio, ya que para evitar que desde la escuela lo memorístico sea lo principal, lo espera- 
ble es que los futuros docentes estén formados en cuanto a pensamiento crítico y analítico-interpretativo como parte de las competencias propias de todo docente.

Parece ser que el marco teórico es el gran incomprendido en la investigación. El verdadero sentido de esta parte del informe de investigación empieza justo al momento en que este se reconoce como una suerte de encrucijada de saberes en el cual se conjuga tanto los aportes teóricos de los autores consultados, la actualidad de lo que se está trabajando e indudablemente del análisis y el argumento que el propio investigador imprima a ese marco teórico. Visto así, no pareciera tener mayores confusiones, sin embargo, el nivel universitario exige ciertos estándares de complejidad en ocasión de reflejar por escrito todo lo anterior.

Además de esto, se ha visto que no se cuenta con un criterio único con respecto a las partes que han de componer el marco teórico aunado a la experticia investigativa que pueda tener la persona encargada de acompañar al estudiante en lo que puede ser la primera o consecutiva experiencia investigativa, lo cual podrá dibujar la ruta por seguir.

Por otra parte, en la formación docente generalmente se espera que se puedan sentar las bases de un futuro profesional que no solo forme parte activa del hecho educativo, sino que pueda proponer y debatir ideas y argumentos desde su autorreconocimiento como sujeto de la educación, con lo cual, es evidente que la experiencia de la investigación científica puede convertirse en un excelente punto de partida para que esa acción transformadora pueda formarse y continuarse ya en la práctica docente en el aula.

Es imperativo el hecho de asegurar que en la formación docente, todo lo que se logre aprender durante el tránsito por los estudios universitarios adquiera su sentido y relevancia. Sin embargo, es de reconocer que las actividades diseñadas para asegurar el aprendizaje de los futuros docentes deben tener su utilidad pero de forma vinculada, es decir, se ha de avanzar en cuanto a la complejidad de las actividades y evidentemente, en el hábito del cuidado de las formas propias de un contexto tanto universitario como profesionalizante de forma que, cuando llegue el momento en que los estudiantes se encuentren con sus respectivos trabajos de fin de sus estudios, la síntesis de los co- nocimientos pueda ser una realidad, en lugar de considerar esos trabajos finales como un requisito más por cumplir, el cual no pocas veces resulta una experiencia no tan satisfactoria. Parece ser que la clave puede estar en lo que pueda asegurarse desde el principio de los estudios evitando numerosas actividades superficiales en detrimento de un sabio equilibrio entre el número de estas y su grado de complejidad.

La habilidad investigativa debe educarse y ella tiene que ver con las acciones que se desarrollan para hacerle frente a los requerimientos que, en este caso, están presentes en las tareas de corte investigativo. Esta noción tiene una serie de implicaciones tanto curriculares como didácticas, pero, en definitiva, se ha de contar con el compromiso del estudiante ante sus estudios docentes.

La gran pregunta es: al tratarse de la formación docente ¿Por qué cuesta tanto reflejar por escrito el resultado de la reflexión y el análisis crítico fundamentado, en el marco teórico de una investigación? Para intentar responder a esta interrogante seguramente se necesiten revisar muchas variables que influyan en esto, sin embargo, se intentarán presentar algunas claves que puedan tenerse en cuenta al momento de abordar esta discusión.

En primer lugar, reconocer que la balcanización curricular tiene sus efectos en el desarrollo de los estudios. Esto significa que la polarización disciplinaria que está presente en los estudios universitarios trae como consecuencia que se observe el dominio teórico de las disciplinas o campos de estudio que conforman el entramado curricular como piedra angular del aprendizaje, obviando el hecho de que la capacidad para saber explicar, debatir, generar pensamiento crítico y analítico pase prácticamente desapercibida en la mayoría de las actividades formativas favoreciendo la realización de tareas de corte mecánico o memorístico. Como se ha podido puntualizar en algún momento de este artículo, lo memorístico será contraindicado solo si se considera como única estrategia para asegurar el aprendizaje.

En virtud de lo anterior, y aunado a los aspectos formales que deben acompañar en este caso la argumentación expresada por escrito, no tendría mucho sentido para el estudiante descubrir estos aspectos prácticamente al final de los estudios y de allí surja la idea de desconocer la inutilidad de 
esto, especialmente al momento de hacer un balance entre lo que se espera en cuanto a la formación docente y lo que se necesita para serlo en la práctica. Se ha de tener en cuenta que lo correcto es evaluar la realización de los objetivos, no de los contenidos.

Resulta necesario que desde las propias universidades se impulsen diseños curriculares que estén armonizados con el objeto de que las competencias en este caso, analíticas y critico-reflexivas puedan formar parte sustancial del desarrollo de los estudios. Esto, traducido en propuestas didácticas favorecedoras de lo anterior, para lo cual entra en juego la formación dirigida al docente universitario, ya que tener experiencia investigativa no necesariamente es sinónimo de saber enseñar a investigar, argumentar o explicar. Es fundamental aunar esfuerzos, unificar criterios y tener presente que, aun contando con innovaciones tecnológicas en el contexto educativo, sin una capacidad argumental fundamentada que sea capaz de analizar, interpretar e inferir, no se podrá obtener aquella habilidad para proponer mejoras en el contexto educativo como resultado de esa experiencia de indagación y búsqueda sistemática y organizada que llamamos investigación.

Continuando con el rol de las universidades, también se hace necesario distribuir con criterio pedagógico el número de estudiantes por atender al momento de emprender la realización de los trabajos finales. Esto con el fin de asegurar una mejor personalización de la acción tutorial que evidentemente es determinante para los estudiantes que se inician o están desarrollando sus primeras experiencias investigativas.

La continuidad curricular puede posicionarse como una gran oportunidad al momento de revisar las posibles alternativas en relación con la mejora de los procesos investigativos de los estudiantes universitarios. Esto quiere decir que, si se sigue viendo la elaboración de los trabajos finales como un requisito engorroso y poco útil para alcanzar una titulación, evidentemente no se podrá apreciar el valor real de todo lo que conlleva esta actividad formativa. Todo esto podrá mejorarse en cuanto se diseñen propuestas curriculares que giren más hacia el beneficio del propio estudiante y menos hacia la adquisición de un conjunto de competencias que no siempre resultan relevantes.
Los cambios en el contexto educativo suelen ser lentos, progresivos y deben ser programados. Sin embargo, lejos de proyectar cambios para el estudiante, más bien proyectar cambios con los estudiantes de forma que se superen las declaraciones loables que pretenden instaurar un nuevo modelo pedagógico universitario centrado en el estudiante, mientras se sigue exigiendo al profesorado universitario producción científica en detrimento de una efectiva y pertinente formación didáctica enfocada exclusivamente al quehacer universitario. En síntesis, las habilidades investigativas ya deben formar parte de la cotidianidad de los estudios universitarios. Por una parte, supone y exige una actitud madura de los estudiantes y por la otra, un compromiso de las universidades $\mathrm{y}$ de los propios docentes universitarios para que se confirme el compromiso adquirido ante la sociedad. Los cambios en la universidad no van a venir desde las publicaciones o desde las declaraciones institucionales, sino desde el reconocimiento de todo lo que supone ser una universidad plural, socialmente pertinente y engarzada con la realidad.

\section{Referencias}

Aguilar-Parra, J., Alías-García, A., Álvarez, J., Fernández-Campoy, J., Pérez-Gallardo, E., \& Hernández, A. (2015). Necesidades de formación del profesor universitario en competencias relacionadas con la acción tutorial. REDU: Revista de Docencia Universitaria, 13(3), 357 375. https://doi.org/10.4995/redu.2015.5433

Alcívar, M., \& Gorozabel, T. (2018). Las tutorías: Percepciones de los estudiantes y docentes universitarios. En Avances en democracia y liderazgo distribuido en educación: Actas del II Congreso internacional de liderazgo y mejora de la educación. Red de Investigación sobre Liderazgo y Mejora de la Educación (RILME). Recuperado de https://bit.ly/2YTA803

Amarillo, E., Corredor, D., \& Ávila, M. (2016). Pensamiento crítico: reflexiones en torno a su importancia para la autonomía. Educación y ciencia, (19), 205-2017. Recuperado de https://bit.ly/2P1HY3c

Bezanilla-Albisua, M., Poblete-Ruiz, M., Fernández-Nogueira D., Arranz-Turnes, S., \& Campo-Carrasco, L. (2018). El Pensamiento Crítico desde la Perspectiva de los 
Docentes Universitarios. Estudios pedagógicos (Valdivia), 44(1), 89-113. https://doi.org/10.4067/S071807052018000100089

Caneiro, M. (2015). Desarrollo curricular y asesorías pedagógicas universitarias. InterCambios. Dilemas y transiciones de la Educación Superior, 2(2), 18-25. Recuperado de https://bit.ly/2Havznd

Carbonell, J. (2017). Pedagogías del siglo XXI. Barcelona: Octaedro.

Davini, M. (2015). La formación en la práctica docente. Buenos Aires: Paidós.

Di Marco, R. (2015). En busca del origen del conocimiento: el dilema de la realidad. Praxis 11(1), 150-162. https:// doi.org/10.21676/23897856.1561

Espinel, N., \& Valbuena, E. (2017). ¿Qué contenidos de biotecnología enseñar en la escuela? Propuesta de una hipótesis de progresión. Biografía 10(19), 448-460. https://doi.org/10.17227/bio-grafia.extra2017-7135

Fernández, C., \& Villavicencio, C. (2017). Desarrollo de habilidades investigativas en la educación superior. Academo, 4(1), 1-12. Recuperado de https://bit.ly/2N6dMBj

Gamboa, M. (2017). Estudio de caso como estrategia didáctica para el proceso de enseñanza-aprendizaje: retos y oportunidades. Bio-grafía 10(19), 1533-1540. https:// doi.org/10.17227/bio-grafia.extra2017-7334

García, E., Porlán, R., \& Navarro, E. (2017). Los fines y los contenidos de enseñanza. En R. Porlán (coord.), Enseñanza universitaria. Cómo mejorarla (pp. 56-72). Madrid: Morata.

García, M. (2017). Desarrollo del pensamiento crítico a través del currículo crítico. Revista Digital Palabra, 3(1), 4857. Recuperado de https://bit.ly/2POOiYT

Gil-Galván, R. (2018). El uso del aprendizaje basado en problemas en la enseñanza universitaria. Análisis de las competencias adquiridas y su impacto. Revista mexicana de investigación educativa, 23(76), 73-93. Recuperado de https://bit.ly/2MnB2es

Gómez, M., Galeano, C., \& Jaramillo, D. (2015). El estado del arte: una metodología de investigación. Revista Colombiana de Ciencias Sociales, 6(2), 423-442. https:// doi.org/10.21501/22161201.1469

Guadarrama, P. (2016). Dirección y asesoría de la investigación científica. Madrid: Docta ediciones.

Guerrero, M. (2007). Formación de habilidades para la investigación desde el pregrado. Acta colombiana de psicología, 1O(2), 190-192. Recuperado de https://bit. ly/2KCKZmp

Guerrero, M. (2016). La investigación cualitativa. INNOVA research journal, 1(2), 1-9. https://doi.org/10.33890/ innova.v1.n2.2016.7

Guevara, R. (2016). El estado del arte en la investigación: ¿análisis de los conocimientos acumulados o indagación por nuevos sentidos? Folios, (44), 165-179. https://doi. org/10.17227/01234870.44folios165.179

Herrera, J., Guevara, C., \& Munster, C. (2015). Los diseños y estrategias para los estudios cualitativos. Un acercamiento teórico-metodológico. Gaceta Médica Espirituana, 17(2), 1-14. Recuperado de https://bit.ly/2YO8aTk

Martín del Pozo, R., Pineda, J., \& Duarte, O. (2017). La formación docente del profesorado universitario. En R. Porlán (coord.), Enseñanza universitaria. Cómo mejorarla (pp. 23-36). Madrid: Morata. https://doi.org/10.4067/ S0718-50062017000100002

Martínez, D., y Márquez, D. (2014). Las habilidades investigativas como eje transversal de la formación para la investigación. Tendencias pedagógicas, (24), 347-360. Recuperado de https://bit.ly/2H71Uer

Martínez, M. (2016). La formación inicial de los maestros: una responsabilidad compartida. Bordón. Revista de pedagogía, 68(2), 9-16. https://doi.org/10.13042/Bordon.2016.68201

Maya, E. (2014). Métodos y técnicas de investigación. Una propuesta ágil para la presentación de trabajos científicos en las áreas de arquitectura, urbanismo y disciplinas afines. México: Universidad Nacional Autónoma de México. Recuperado de https://bit.ly/2nysQcq

Mero, J. (2019). Aplicación de las destrezas con criterio de desempeño para el desarrollo de la comprensión lectora. Rehuso, 4(1), 76 - 86. https://doi.org/10.33936/rehuso. v4i1.1683

Morales-Gómez, G., Arteaga-Rolando, M., Gallegos-Samaniego, A., Yanchapaxi-Sánchez, N., \& Stay-Zúñiga, J. (2016). Tendencias metodológicas universitarias en lossiglos XX y XXI. Estudio comparativo. Revista Ciencia Unemi, 9(17), 125-135. https://doi.org/10.29076/issn.2528-7737vol9iss17.2016pp125-135p

Moreno-Pinado, W., \& Velázquez, M. (2017). Estrategia didáctica para desarrollar el pensamiento crítico. REICE. Revista Iberoamericana sobre Calidad, Eficacia y Cambio en Educación, 15(2), 53-73. https://doi. org/10.15366/reice2017.15.2.003

Moyano, E. (2018). La enseñanza de la lectura y la escritura académicas mediante programas a lo largo del curriculum universitario: opción teórica, didáctica y de gestión. DELTA: Documentação de Estudos em Lin güística Teórica e Aplicada, 34(1), 235-267. https://doi. org/10.1590/0102-445074896274115057 
Ochoa, L., \& Cueva, A. (2014). El plagio y su relación con los procesos de escritura académica. Forma y función, 27(2), 95-113. https://doi.org/10.15446/fyf.v27n2.47667

Ossa-Cornejo, C., Palma-Luengo, M., Lagos-San Martín, N., Quintana-Abello, I., \& Díaz-Larenas, C. (2017). Análisis de instrumentos de medición del pensamiento crítico. Ciencias Psicológicas, 11(1), 19-28. https://doi. org/10.22235/cp.v11i2.1343

Palacios, W., Álvarez, M., Moreira, J., \& Morán, C. (2017). Una mirada al pensamiento crítico en el proceso docente educativo de la educación superior. Revista Educación Médica del Centro, 9(4), 194-206. Recuperado de https://bit.ly/2KRTw3C

Quevedo, N., García, N., \& Cañizares, F. (2018). La habilidad de interacción investigativa entre tutor y tutorado. Revista Magazine de las Ciencias. 3(2), 117-130. Recuperado de https://bit.ly/2z53ZU4

Ramírez-Casallas, J. (2018). Hipótesis y criterios de trabajo en torno a las intervenciones que potencian la Reforma Curricular. Medellín: Universidad Cooperativa de Colombia. https://doi.org/10.13140/ RG.2.2.33306.13764Risco del Valle, E. (2018). Recurrencias argumentativas y cuestiones en trabajos de titulación de pregrado: insumos para la programación de la formación argumental a nivel de pregrado. Revista Praxis Pedagógica, 18(22), 18-48. http://doi. org/10.26620/uniminuto.praxis.18.22.2018-48

Rivadeneira, E. (2017). Lineamientos teóricos y metodológicos de la investigación cuantitativa en ciencias sociales. In Crescendo, 8(1), 115-121. https://doi.org/10.21895/ incres.2017.v8n1.11

Rivero, A., \& Porlán, R., (2017). La evaluación en la enseñanza universitaria. En R. Porlán (coord.), Enseñanza universitaria. Cómo mejorarla (pp. 73-91). Madrid: Morata.

Rodríguez, A., \& Pérez, A. (2017). Métodos científicos de indagación y de construcción del conocimiento Revista EAN, 82, pp.179-200. https://doi.org/10.21158/01208160. n82.2017.1647
San Martín, D. (2014). Teoría fundamentada y Atlas. ti:recursos metodológicos para la investigación educativa. Revista electrónica de investigación educativa, 16(1), 104-122. Recuperado de https://bit.ly/2LQkck5

Santibáñez, C. (2014). ¿Para qué sirve argumentar?: Problematizando teórica y empíricamente el valor y la función de la argumentación. Círculo de lingüística aplicada a la comunicación, 58, 163-205. https://doi.org/10.5209/ rev_CLAC.2014.v58.45474

Serrano, R., \& Pontes, A. (2015). Nivel de desarrollo de las competencias y objetivos generales del Máster Formación del Profesorado de Enseñanza Secundaria. Perfiles educativos, 37(150), 39-55. https://doi.org/10.22201/ iisue.24486167e.2015.150.53161

Tamayo, O., Zona, R., \& Loaiza, Y. (2015). El pensamiento crítico en la educación. Algunas categorías centrales en su estudio. Revista Latinoamericana de Estudios Educativos, 11(2) 111-133. Recuperado de https://bit. ly/2X9huws

Tusco, L., Quesocala, V., \& Tito, E. (2011). El marco teórico. Revista de Actualización Clínica Investiga, 10, 466-470. Recuperado de https://bit.ly/3071hsU

Urbina, J. (2016). El arte de aprender con pasión. Cómo aprenden los estudiantes universitarios cuando estudian con pasión. Encuentros, 14(1), 15-29. https://doi. org/10.15665/re.v14i1.666

Venegas-Ramos, L., \& Gairín, S. (2018). Gestión y desarrollo de planes de acción tutorial en la universidad. Estudios de casos. REOP-Revista Española de Orientación y Psicopedagogía, 29(1), 125-143. Recuperado de https://bit.ly/2Z4XfnH

Vidal-Moscoso, D., \& Manríquez-López, L. (2016). El docente como mediador de la comprensión lectora en universitarios. Revista de la educación superior, 45(177), 95-118. https://doi.org/10.1016/j.resu.2016.01.009

Yubero, S., \& Larrañaga, E. (2015). Lectura y universidad: hábitos lectores de los estudiantes universitarios de España y Portugal. El profesional de la información, 24(6), 717-723. https://doi.org/10.3145/epi.2015.nov.03 es un artículo de acceso abierto, distribuido bajo los términos de la LicenciaCreativeCommons Atribución-CompartirIgual 4.0 Internacional.(http://creativecommons.org/licenses/by-sa/4.0/), que permite el uso no comercial, distribución y reproducción en cualquier medio, siempre que la obra original sea debidamente citada. 